\title{
Heating Systems Based on Thermal Generators with the Effect of Ranke
}

\author{
Oleg Zaytsev, Liliy Petrekevish, Nataliy Bogatikova, Igor Perminov \\ National Academy of Environmental and Resort Construction \\ Chair "Heat, Gas supply and Ventilation" \\ e-mail: zon@ukr.net
}

\begin{abstract}
Results on the distribution of velocity, temperature and pressure in the proposed design of the vortex chamber. The effect of Ranke in this way of burning gas and a method for regulating heat removal in double-circuit boilers of small capacity.
\end{abstract}

Key words: furnace, gas flaring, the vortex effect

\section{Maintenance}

The current state of fuel and energy complex of the country requires urgent action on research, development and implementation of fuel sources. The bulk of these sources must be in the country. The solution to this problem is possible if the orientation of the heating complex for use of recycled and renewable sources of energy and efficient use of fossil fuels [1-3]. Currently, the energy crisis affects not only heavy industry but also the domestic economy, especially because of the value of energy consumption of these areas of the economy is already comparable.

The main trends in western Europe North America heat-generating technology is the development and introduction of low-power boilers operating at non-traditional, low-energy, renewable fuels [4]. However, these boilers are characterized by a low efficiency, but due to the fact that the choice of power such equipment is carried out on the value of heat loss in cold five days, they are working off-design mode, ie with lower efficiency [5]. This increases the emission of harmful substances into the atmosphere - that was the reason for the use of such facilities only in agricultural areas (in most countries their use is prohibited in urban areas). The rare use of known designs of heating boilers of small capacity with highly accelerated firing process due to the obscurity of this issue from both theoretical and practical point of view $[6,10]$. 


\section{The main result of}

Nature of the motion of two-phase flow in the combustion process in the furnace is determined by the simultaneous action of the following major factors [7]:

- Dynamic effects of the gas jet on the torch burning;

- Effect of centrifugal force and gravity on the temperature field in the furnace;

- Combustion of fuel in the combustion space;

- The process of removal of heat from the device.

In this case, the technological conditions of the combustion process imposes constraints on the temperature distribution in the device and the value of certain temperature zones. Thus, to determine the optimal parameters (speed, flow, temperature in the unit) with full fuel combustion and maximum use of funds in the furnace heat is necessary to investigate the laws of aerodynamics the furnace under the influence of these factors [8-10].

However, due to the fact that the analysis of the literature revealed a lack of adequate description of the process under study of existing mathematical methods to solve this problem, we used specialized software package [6]. In this task, the motion of a fluid is modeled using the Navier - Stokes equations describing the unsteady formulation in the laws of conservation of mass, momentum and energy of this environment. In addition, use the equations of state of the components of the fluid, as well as empirical dependence of the viscosity of the medium components on the temperature. These equations are simulated turbulent, laminar and transitional (between laminar and turbulent, the transition is determined by the critical value of Reynolds number) flows. To simulate the turbulent flow (in this case) of the Navier - Stokes equations are averaged according to Reynolds, that is, used averaged over a small time-scale effects of turbulence on the flow parameters, and largescale temporal variations averaged over a small time-scale components of gas-dynamic flow parameters (pressure, velocity, temperature) are taken into account by introducing appropriate time derivatives. As a result, the equations have additional terms - for the Reynolds stress, and for the closure of this system is used the transport equation of turbulent kinetic energy and its dissipation in the turbulence model [6].

This system of equations of conservation of mass, momentum and energy is not stationary spatial flow looks like the approach of Euler in the rectangular coordinate system rotating with angular velocity $Q$ about the axis passing through its origin [6]:

$$
\begin{gathered}
\frac{\partial p}{\partial t}+\frac{\partial}{\partial x_{k}}\left(\rho u_{k}\right)=0 \\
\frac{\partial\left(\rho u_{i}\right)}{\partial t}+\frac{\partial}{\partial x_{k}}\left(\rho u_{i} u_{k}-\tau_{i k}\right)+\frac{. \cdot}{\partial x_{i}}=S_{i} \\
\frac{\partial(\rho E)}{\partial t}+\frac{\partial}{\partial x_{k}}\left((\rho E+P) u_{k}+q_{k}-\tau_{i k} u_{i}\right)=S_{k} u_{k}+Q_{H}
\end{gathered}
$$

where $\mathrm{t}$ - time, $\mathrm{u}$ - velocity of the fluid, $\rho$ - fluid density, $\mathrm{P}$ - pressure of the fluid, $\mathrm{Si}$, - the external mass forces acting on unit mass of fluid:, E - total energy of unit mass of fluid; $\mathrm{Q}_{\mathrm{H}}$ - 
heat from the heat source in a unit volume of fluid; $\tau$ - tensor of viscous shear stress; qi diffuse heat flux; subscripts indicate summation over the three coordinate directions.

For Newtonian fluids the viscous shear stress tensor is defined as follows:

$$
\tau_{i j}=\mu\left\{\frac{\partial u_{i}}{\partial x_{j}}+\frac{\partial u_{j}}{\partial x_{i}}+\frac{2 \partial u_{j}}{3 \partial x_{j}} \delta_{i j}\right\}-\frac{2}{3} \rho k \delta_{i j}
$$

In accordance with the $\mathrm{k}-£$ turbulence model, $\mu$ is determined by the kinetic energy of turbulence $\mathrm{k}$ and the dissipation of this energy $\varepsilon[6]$ :

$$
\mu_{i}=f_{\mu} \frac{C_{\mu} \rho k^{2}}{\varepsilon}
$$

In this model, applied the "internal" problem - that is, air, natural gas is fed through a tangential connections in the central part of the furnace (air and gas flow rate of $0.1 \mathrm{~kg} / \mathrm{s}$, with mass fractions of 0.3 and 0.7 , respectively). The air temperature was set equal to $20^{\circ} \mathrm{C}$, while natural gas and solid particles $-1100^{\circ} \mathrm{C}$. Removal of combustion products was carried out through an annular aperture with one hand and an axial hole on the other side of the device. In this case, to simulate the process of selecting a warm energy through the surface of the wall were set the thermal conductivity of the wall (except for middle area) $-50 \mathrm{~W} / \mathrm{m}^{\circ} \mathrm{C}$ and outside surface temperature $\left(60^{\circ} \mathrm{C}\right)$. Figure 1 shows a general view of the device, which consists of a combustion chamber in which a tangential connections served a mixture of gas and air, and combustion gases through oppositely arranged ring and an axial hole.

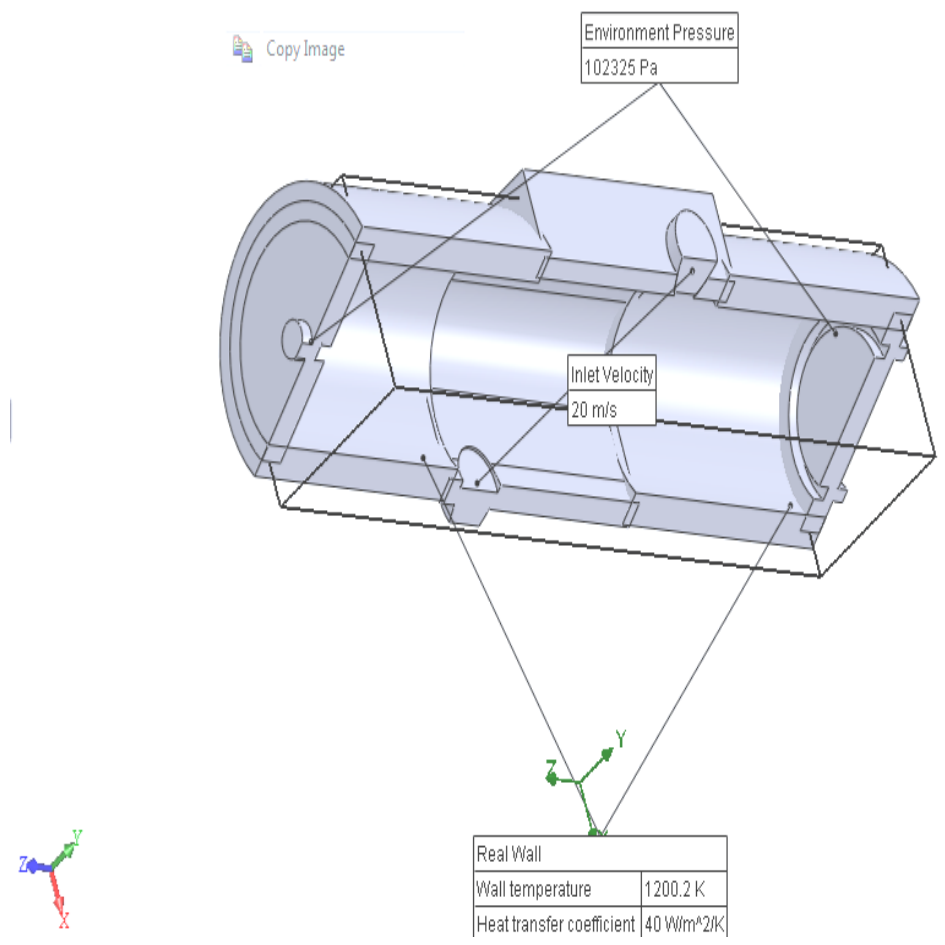

Figure 1: General view of model 
The studies found that when an organization of gas burning in the furnace of a redistribution of flow on temperature, similar to (though to a lesser extent) the distribution of vortex tubes as a result of the development of Ranke. That is a zone of higher temperatures in parts of the structure, where the flue gas through an annular aperture and, consequently, lower temperature zone in the central outlet of the combustion products. At the same time revealed the possibility of adjusting the ratio of these temperature fields proportional sizing of the vents, which, in turn, allows you to control heat removal from the respective surfaces, depending on desired heat consumption for the needs of heating and hot water (respectively for heating the region with the highest temperature zone and hot water - with the lower).

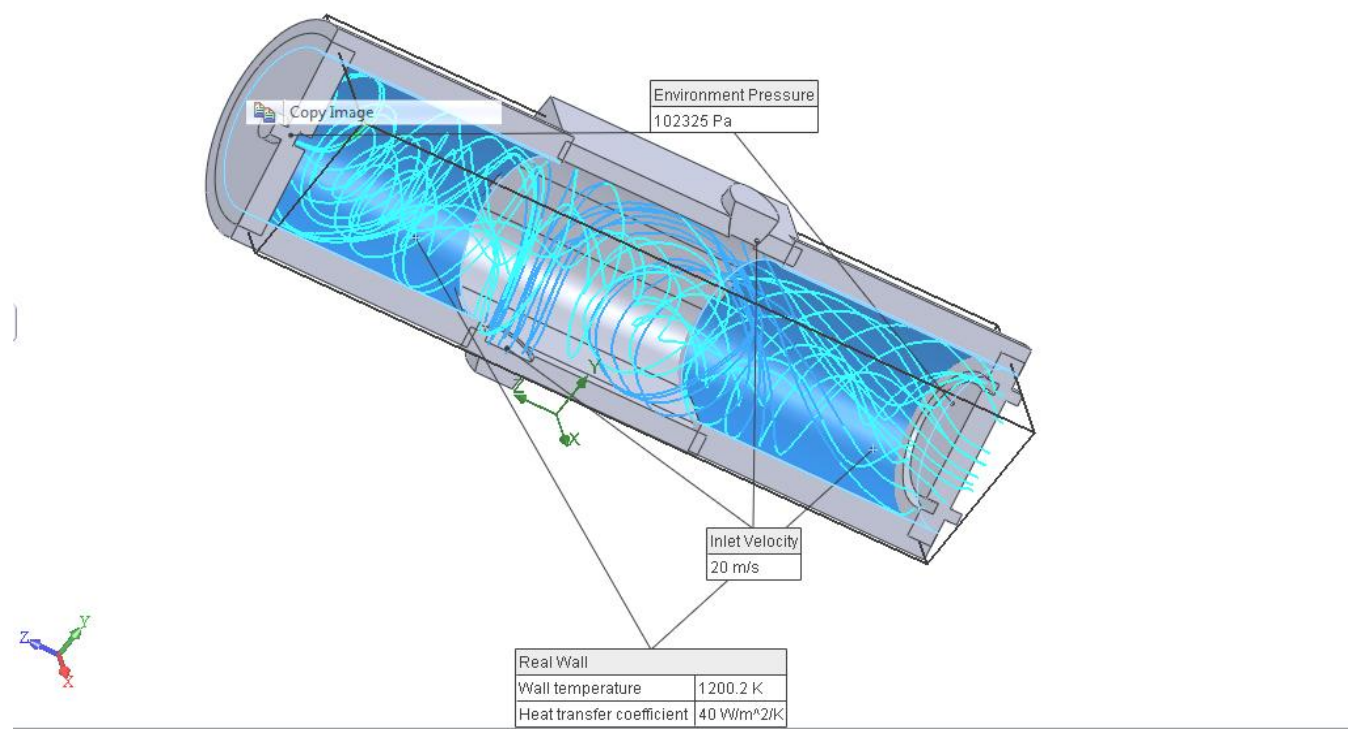

Figure 2: The change in temperature along the current lines

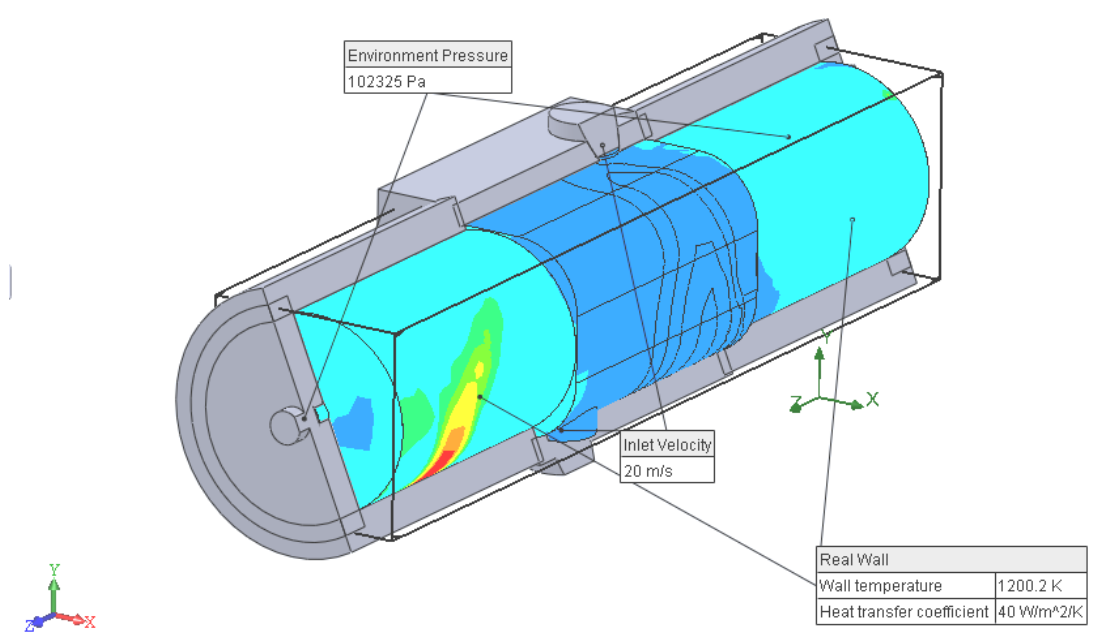

Figure 3: The temperature distribution on the surface of the furnace 
To confirm the theoretical results developed and made a stand which is based on a device for burning gas in a vortex tube [11-13], which works as follows.

Gas-air mixture is fed through two tangential nozzle, and further fell to the device, where the envelopes were burning. Heat removal from the body by means of two external water circuit. Air flow was measured by a diaphragm chamber, calculated by the method, translated in [14], and which is connected to a U-shaped differential manometer. Regulation of air flow was carried out dampers. Measurements of the flow temperature was platinum-rhodium thermocouple, and temperature of the surface - with the help infrokrasnogo thermometer with laser pointer.

Comparing the results of theoretical studies of the interaction of twisted jets of gas combustion in a vortex combustor with experimental dannyami (Fig. 4) confirmed the adequacy of the proposed mathematical models and the justification made by Research opinion, with the discrepancy between the results does not exceed 10\% [14], which allows to use the theoretical relationships in the engineering methods of calculation. On the above photographs (Fig.5-6) with an open axial withdrawal of the redistribution of the flame is similar to the redistribution of velocity in a vortex tube (Fig. 5), while the closed axial withdrawal - the flame covers the entire area of the pipe (Fig. 6).

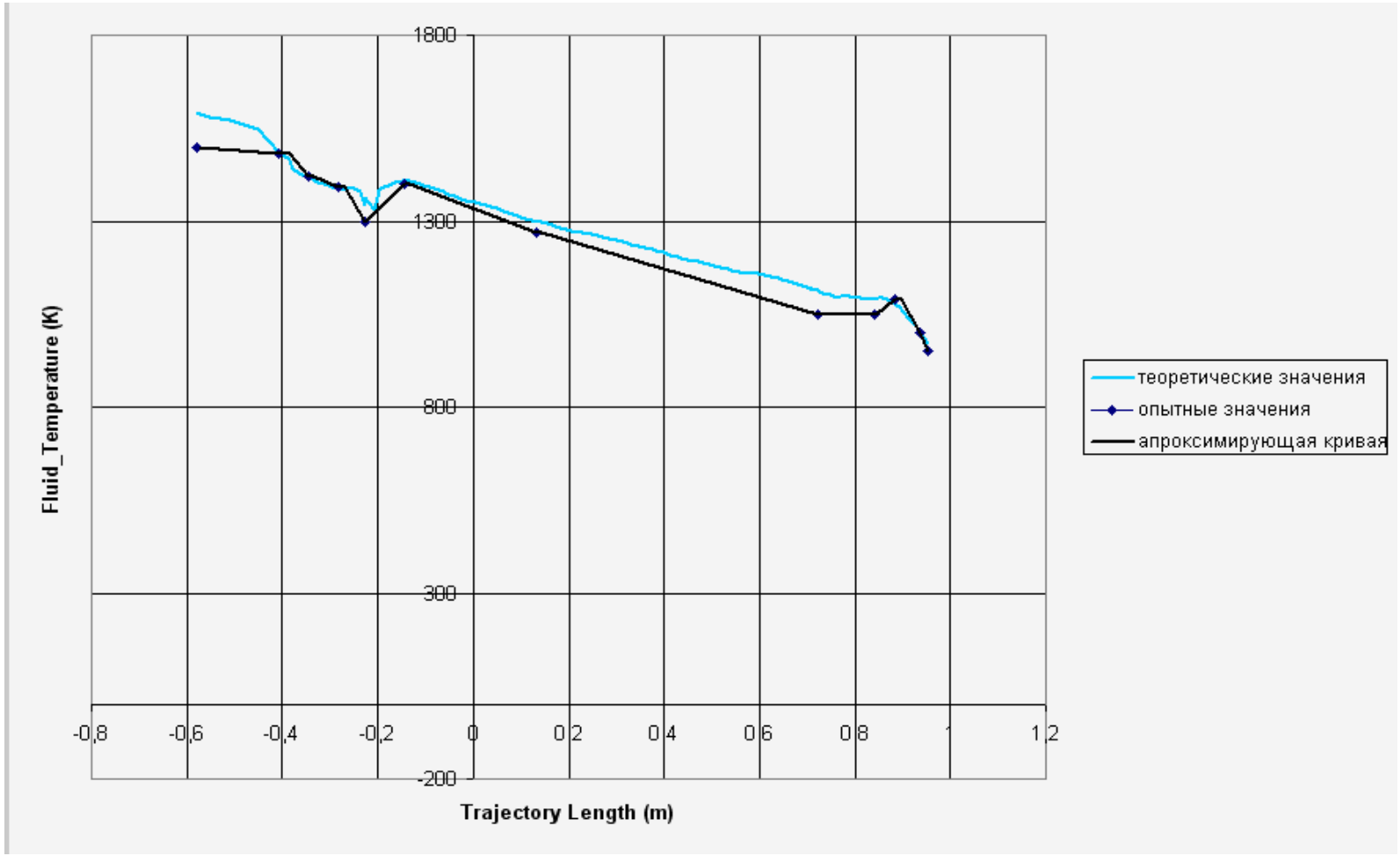

Figure 4: The temperature boiler wall. Experimental and theoretical data. 


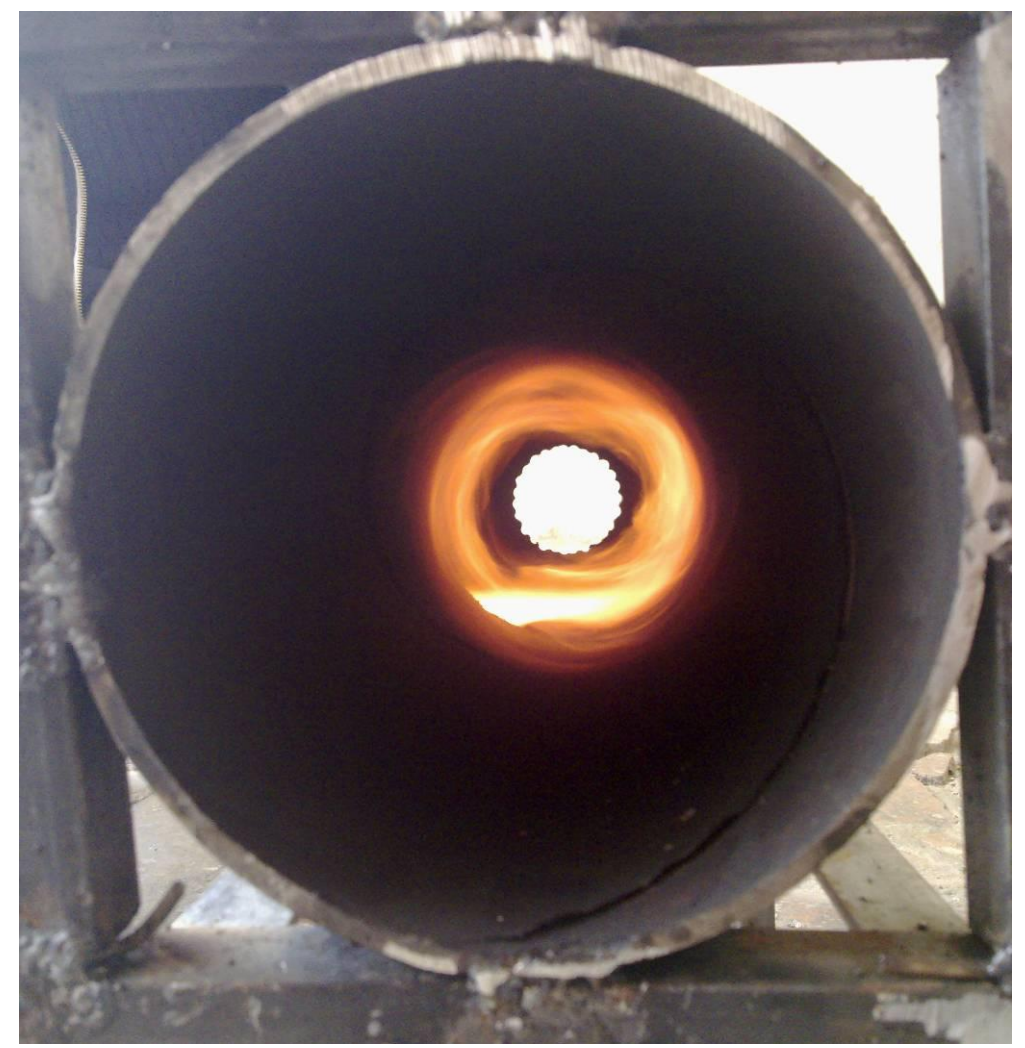

Figure 5: Pilot plant. View from the throttle valve (an axial hole open)

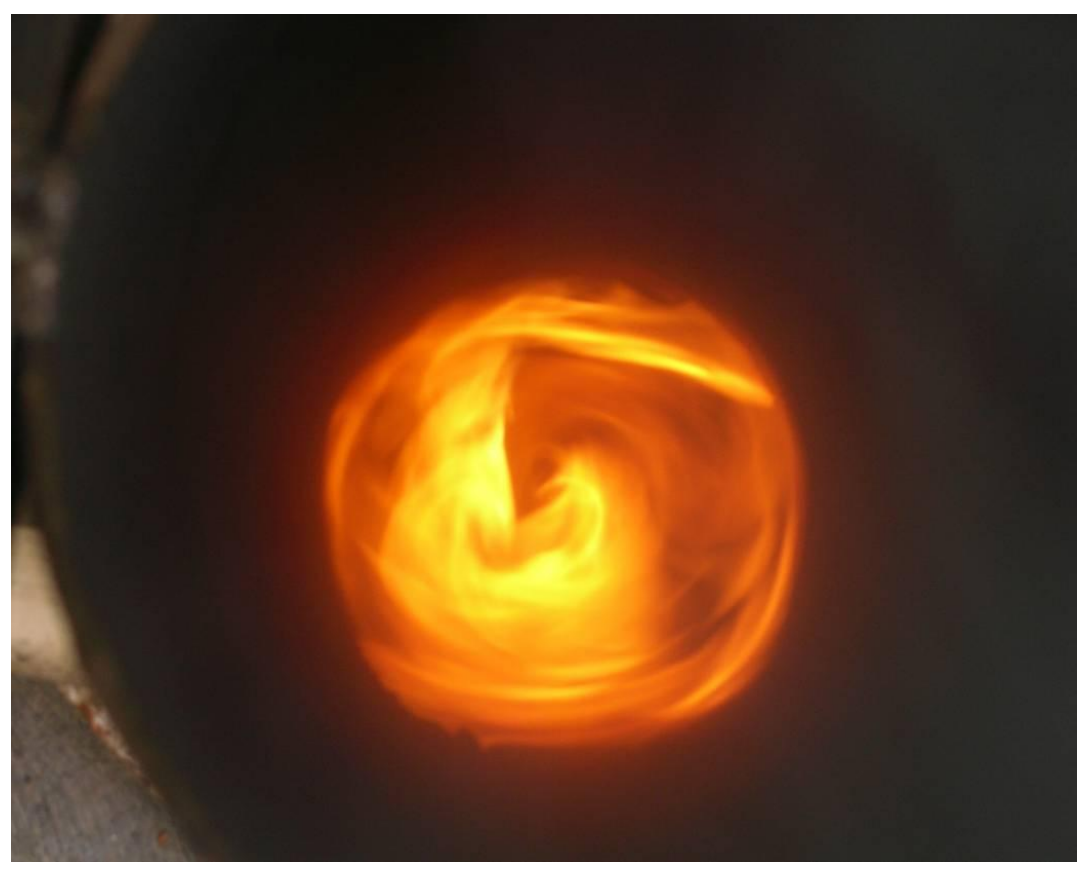

Figure 6 Pilot plant. View from the throttle valve (an axial hole is closed) 


\section{CONCLUSIONS}

1. In the proposed method to gas burning in the firebox is a redistribution of flows on the temperature, a similar distribution in the vortex tubes as a result of the development of Ranke.

2. Identified the zone of higher temperatures in parts of the structure, where the flue gas through an annular aperture and, consequently, lower temperature zone in the central outlet of the combustion products.

3. The possibility of regulating the relations of temperature fields proportional to the change in the vent area, which in turn allows you to adjust the heat removal from the respective surfaces, depending on desired heat consumption for the needs of heating and hot water.

4. Comparing the results of theoretical studies of the interaction of twisted jets of gas combustion in a vortex combustor with experimental dannyami confirmed the adequacy of the proposed mathematical models.

\section{References}

[1] Teplovoy calculation of boilers: a normative method. (1973). Moscow: Energiya.

[2] Deev, L. V. (1990). Boiler installation and maintenance. Deev LV, Balakhnichev NA; practical. allowance. - M.: Vyssh. shk..

[3] Klyuchnikov, A. D. (1990). Heat transfer and thermal conditions in industrial furnaces. Klyuchnikov AD, Kuzmin VN Popov, SK; uch allowance for higher education. - M.: Energoatomizda.

[4] Zaytsev, O. N. (2004). Intensifikatsiya protsesiv vzacmodiï obertovih gas potokiv in energozberegayuchih tehnologiyah teploenergetiki. Doctoral dissertation of Engineering, Odessa, ONPU.

[5] Zaytsev, O. N. (2002). Energy-saving technology in heating systems, decentralized, based on interacting swirling flows. In International Scientific Conference Actual problems of urbanization and spatial construction. Chisinau 2002 (349-354). Moldova.

[6] Alyamovsky, A. A. (2005). SolidWorks. Computer modeling in engineering practice. Petersburg: BHV.

[7] Deych, M. E., Filippov, G. A. (1981). Gas dynamics of two-phase media. - Moskwa: Energoizdat ed th e 2-revised. and add.

[8] Dzhaluriya, I. (1983). Natural convection: Heat and Mass Transfer. Per. from English. Moscow: Mir.

[9] Goldshtik, M. A. (1981). Eddy currents. Novosibirsk: Nauka.

[10] Gaydabura, I. P., Vaganov, A. I., Todortsev, J. K. (1986). Management energotechnological vortex devices using their acoustic field. Industrial Energy. Moscow: Energoatomizdat, 7, 3639.

[11] Danilin, E. A Klochkov, V. N (1988). Control of fuel combustion in industrial boilers. K.: Technique.

[12] Dmitriev, L. S., Kuz'mina, L. V., Moshkarnev, L. M. (1984). Experimental Design in ventilation and air conditioning. Irkutsk: State University. 
[13] Naitdenov, G. F. (1974). Gas burner device with adjustable flame characteristics. K.: Tehnika.

[14] Novitsky, P. V., Zograf, I. A. (1985). Estimation of errors of measurement results. Leningrad: Energoatomizdat. 